\title{
DIFERENCIAS EN EL PROCESAMIENTO SENSORIAL ENTRE NIÑOS/AS PRETÉRMINOY A TÉRMINO: EL PAPEL DEL TERAPEUTA OCUPACIONAL
}

\author{
SENSORY PROCESSING DIFFERENCES BETWEEN PRETERM AND TERM INFANTS: THE \\ ROLE OF THE OCCUPATIONAL THERAPIST
}

\section{Jéssica Romero-Sánchez ${ }^{1}$}

\begin{abstract}
Resumen
En el presente trabajo se comparó un grupo de niños prematuros (menores de 28 semanas de edad gestacional) con un grupo de niños nacidos a término, con el objetivo de valorar cuáles son las disfunciones de integración sensorial de una muestra de niños prematuros de la ciudad de Granada, España, para poder establecer terapias que mejoren estos déficit. Para esto se realizó un estudio en el que utilizaron escalas de valoración validadas para esta población. Atendiendo a los resultados encontrados anteriormente, se esperó encontrar que los niños nacidos a término mostrasen mayores disfunciones en los sistemas sensoriales táctil, vestibular-propioceptivo, y auditivo que los niños nacidos a término. Asimismo, se esperó que los prematuros mostrasen una mayor problemática en el cuadrante de bajo registro que los niños nacidos a término. Para contrastar esta hipótesis se les pidió a los padres de los niños pertenecientes a los dos grupos que contestarán la versión en castellano del Sensory Profile (Dunn, 2014). Los resultados mostraron que, aunque las puntuaciones de ambos grupos se encontraron dentro de los valores considerados como valores norma en el Sensory Profile, los niños prematuros, en comparación con el grupo control, obtuvieron una puntuación significativamente más alta en el cuadrante sensorial de bajo registro, y en los estilos sensoriales de movimiento y tacto. Estos resultados sugieren que lo niños pretérmino podrían beneficiarse de un tratamiento en integración social realizado por terapeutas ocupacionales especializados.2
\end{abstract}

\section{Palabras clave:}

Nacimiento prematuro, niño prematuro, desorden sensorial.

\begin{abstract}
In this paper we compared a group of preterm infant (less than 28 weeks of gestational age with a control group to investigate sensory integration dysfunctions in a sample of preterm infants of Granada, Spain, in order to highlight the importance of specific therapies that could improve such disorders. Building on previous results, we expected to find that preterm infants will show greater dysfunctions in the sensory tactile and the vestibular-proprioceptive, and auditory systems, compared to children born at term. We also hypothesized that preterm infants will show greater problems in the low registration quadrant. To examine these ideas we asked the children's parents to answer the Spanish version of the Sensory Profile (Dunn, 2014). Results shown that, although both groups (term and preterm) exhibited normative values accordingly to the Sensory Profile, preterm infants, compared to the control group, scored significantly higher in low registration quadrant, and in the tactile and movement styles. These results suggest that preterm infants might benefit from a sensory integration treatment, carried out by a specialized occupational therapist.
\end{abstract}

\section{Key words:}

Preterm birth, premature infants, disorders of sensory processing.

1 Terapeuta Ocupacional.Centro de Atención Temprana de San Rafael de Granada

Profesora Asociada Departamento de Fisioterapia (Área Terapia Ocupacional) Universidad de Granada Dirección Postal: C/ Jesús y María 11, tercero. 18009. Granada Teléfono: 636208103 Correo electrónico: jessicaromero82@hotmail.com 


\section{INTRODUCCIÓN}

Los niños prematuros - aquellos nacidos antes de las 37 semanas de edad gestacional-, en especial la población conocida como muy prematuros - nacidos con una edad gestacional entre 34 y 30 semanas-y prematuros extremos - nacidos entre las 29 y las 26 semanas-, presentan con una elevada frecuencia disfunciones de integración sensorial (Bart, Shayevits, Gabis, \& Morag, 2011; Mitchell, Moore, Roberts, Hachtel, \& Brown, 2015; Rahkonen et al., 2015; Wickremasinghe, Rogers, Johnson, Shen, Barkovich, y Marco, 2013). En este artículo se presenta un estudio que en el que se investigan estas diferencias en una muestra de niños y niñas prematuros de la ciudad de Granada, España.

A continuación se presentará un panorama de la situación de la atención temprana y la terapia ocupacional en esta ciudad y una breve introducción a la teoría de integración sensorial, para posteriormente plantear la pregunta de investigación en la que el presente trabajo se focaliza.

\section{Atención Temprana y Terapia Ocupacional}

Este estudio se realiza dentro del marco de la situación que vive la atención temprana en España; en concreto, en la ciudad andaluza de Granada. Es necesario hacer esta concreción debido a las grandes diferencias existente entre los recursos de los centros de las diferentes comunidades, ya que no existe un marco legislativo nacional. (Domínguez y Calvo, 2015).

Los centros de atención temprana en España surgen en los años 70 como centros de Estimulación Precoz, donde la atención se dirige fundamentalmente a la intervención en la niñez. Actualmente, la intervención es concebida de una forma más global y se dirige a la niñez, a la familia y a la comunidad. Además, en el año 2000, y gracias al trabajo de los profesionales que constituyen la Federación Estatal de Asociaciones de Profesionales de Atención Temprana (GAT), se publicó el Libro Blanco de atención temprana, como documento base para la planificación y coordinación del servicio de atención temprana (Domínguez y Calvo, 2015). En este documento se define la atención temprana como "el conjunto de intervenciones dirigidas a la población infantil de 0-6 años, a la familia y al entorno, que tienen por objetivo dar respuesta lo más pronto posible a las necesidades transitorias o permanentes que presentan los niños con trastornos en su desarrollo o que tienen el riesgo de padecerlos. Estas intervenciones, que deben considerar la globalidad del niño, han de ser planificadas por un equipo de profesionales de orientación interdisciplinar o transdisciplinar" (AAVV. 2000, p. 12). Dado que la terapia ocupacional se encarga de "la utilización terapéutica de las actividades de la vida diaria, productivas y de ocio en personas que se encuentran limitadas (...), con los fines de maximizar la independencia, prevenir la discapacidad, mejorar el desarrollo y mantener la salud. Se incluye la adaptación de tareas y/o la intervención sobre el ambiente para lograr la máxima independencia y mejorar la calidad de vida" (Asociación Profesional de Terapeuta Ocupacionales de Navarra, 2004, párrafo 3), queda sobradamente justificada la presencia del terapeuta ocupacional dentro de este equipo multidisciplinar, ya que ésta permite aumentar el potencial individual de cada niño/a —en los distintos contextos en los que se desenvuelve- a través del uso de actividades terapéuticas.

No obstante, solo el $1.14 \%$ de todos los profesionales que componen estos equipos multidisciplinares de atención temprana son terapeutas ocupacionales (Domínguez y Calvo, 2015). Asimismo, cabe destacar que pocos de estos terapeutas ocupacionales cuentan con formación en integración sensorial (ya que esta comenzó a hacerse de manera extensiva en España a partir de 2008, año en que Erna Imperatore comenzó a impartir los cursos de formación en integración sensorial reconocidos por la Universidad del Sur de California).

\section{La Teoría de la Integración Sensorial}

La Teoría de la integración sensorial fue propuesta por la terapeuta ocupacional Jean Ayres (1978), quien definió la integración sensorial como el "proceso neurológico que organiza las sensaciones del propio cuerpo y del medio ambiente, y hace posible usar el cuerpo efectivamente en el entorno". La teoría de la integración sensorial describe: el procesamiento normal de la información sensorial, el desarrollo sensorial normal en los niños y las disfunciones de integración sensorial (Moral, Pastor, y Sanz, 2013).

Al hablar de disfunciones en la integración sensorial, se hace referencia a las disfunciones en "la capacidad del Sistema Nervioso Central para organizar e interpretar las informaciones captadas por los diversos sistemas sensoriales (visual, auditivo, gustativo, olfativo, táctil, propioceptivo y vestibular) y poder responder así de forma adecuada al ambiente que nos rodea" 
(Asociación Española de Integración Sensorial, s.f.). Así, mientras que el proceso normal consiste en percibir los estímulos del propio cuerpo o del entorno, organizar la información percibida y responder de manera adecuada a las demandas de dicha información, muchos niños tienen dificultades a la hora de seguir esta secuencia lógica, enfrentándose a la disfunción de integración sensorial (DIS) o desorden en el procesamiento sensorial (DPS) (Imperatore-Blanche, 2005). Esta disfunción de integración sensorial puede afectar a cualquiera de los sistemas sensoriales del niño e impactar así en su funcionamiento cotidiano (Imperatore-Blanche, 2005).

Figura 1.

Propuesta de una nueva nosología de los desórdenes de procesamiento sensorial. Adaptada de Miller y cols. 2007.
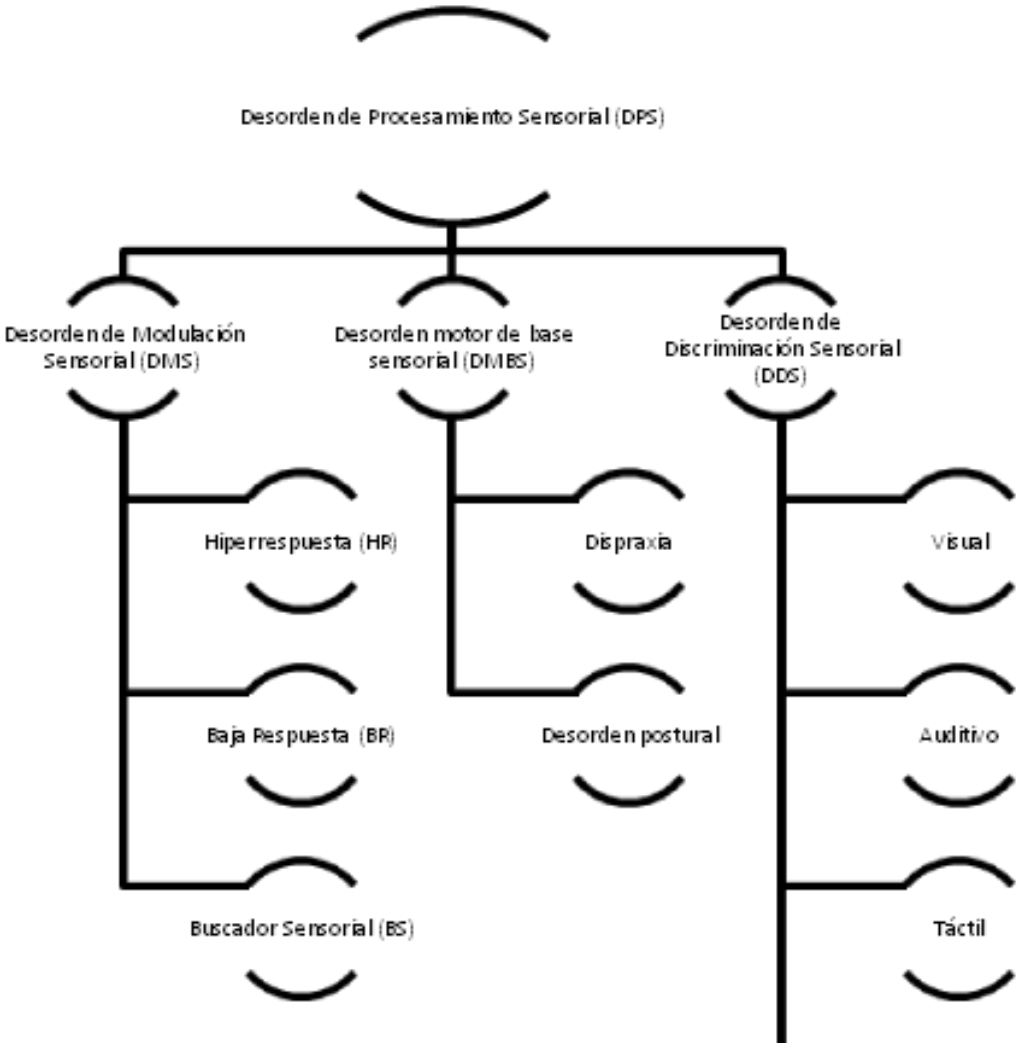

Desde la Teoría de la integración sensorial, y en relación a los desórdenes de procesamiento sensorial, Miller y cols. (2007) proponen una clasificación agrupando dichos desórdenes en tres grupos: desórdenes de modulación, desórdenes motores de base sensorial y desórdenes de discriminación (véase Figura 1). Es necesaria esta nueva propuesta nosológica ya que aporta una mayor especificidad diagnóstica, lo que mejorará la homogeneidad de los ejemplos usados para la investigación empírica, promoviendo así unos objetivos de intervención más ajustados a los subtipos de diagnósticos específicos (Miller y cols., 2007).
Así, dentro de los desórdenes de modulación encontramos al niño hiper-responsivo, al niño de baja respuesta y al niño buscador sensorial (Miller y cols., 2007). El niño hiperresponsivo puede actuar de dos manera, o bien evitando completamente el estímulo (evitador sensorial) o bien respondiendo al input sensorial más rápido y con mayor intensidad.

Por su parte, el niño hiperresponsivo es aquel que puede parecer más rápido que el resto, que presenta una respuesta más intensa o exagerada a los estímulos, cambios de ambiente o transiciones entre actividades. Ade- 
más, puede mostrar un amplio rango de comportamientos, desde muy activo a más pasivo. (Miller y cols., 2007)

El niño baja respuesta o hiporresponsivo, en cambio, parece no percibir los estímulos del ambiente que le rodea, lo que puede provocarle una falta de interés por explorar o interrelacionarse con otros, pudiendo aparecer apático. Suelen reaccionar poco al dolor, temperatura y parecen no notar cuando se ensucian. (Miller y cols., 2007)

Finalmente, el niño buscador sensorial buscar fuertemente los estímulos que prefiere (de tipo motor, auditivo, visual, alimentos, etc.) dentro del ambiente que le rodea. Suele parecer que tiene poca conciencia del peligro. Parecen impulsivos, desorganizados y les cuesta completar las actividades que se le pide que realices si ellos no las han elegido o no se encuentran dentro de sus preferencias. (Miller y cols., 2007).

\section{Integración Sensorial y Prematurez}

En diversos estudios seha encontrado que los niños nacidos a prétermino suelen presentar mayores problemas de integración sensorial que los niños nacidos a término (Bart et al., 2011; Wickremasinghe et al., 2013, Rahkonen et al., 2015)3,22]]\}\}\}, ‘"id":1079," uris":["http://zotero.org/users/1726773/items/FAE2T5CH"],"uri":["http:// zotero.org/users/1726773/items/FAE2T5CH"],"itemDa ta":\{“id":1079,"type":"article-journal","title":"Atypical sensory processing is common in extremely low gestational age children","container-title":"Acta Paediatrica"," page":"n/a-n/a","source":"Wiley Online Library","abstract":"Aim $\backslash n \backslash n$ Atypical sensory processing is common in children born extremely prematurely. We investigated sensory processing abilities in extremely low gestational age (ELGA. Por ejemplo, Bart y colaboradores hallaron que los niños nacidos en una edad gestacional de 34-35 semanas mostraban problemas en las áreas táctil, vestibular-propioceptivo y auditivo (Bart et al., 2011), mientras que no encontraron problemas en las áreas visual u oral. Wickremasinghe y colaboradores (2013) replicaron estos resultados utilizando niños nacidos con una edad gestacional menor a 32 semanas. Además, estos autores pusieron en evidencia que el cuadrante más afectado fue el de bajo registro, ya que el $23 \%$ de los niños prematuros examinados mostraron disfunciones en este cuadrante. Por su parte, y utilizando una muestra de prematuros extremos $(<25$ semanas), Rahkonen y colaboradores también hallaron que el cuadrante más afectado era el de bajo registro, evidenciando que un $23 \%$ de los niños prematuros se encontraban afectados (Rahkonen et al., 2015).

Otros autores plantean que los desordenes de procesamiento sensorial que presentan los niños prematuros podrían deberse a las diferencias en la estimulación que han recibido (Mitchell et al., 2015). Por un lado, los prematuros reciben una menor estimulación intra-utero (Graven y Browne, 2008), pero experimentan estímulos de una fuerte intensidad en las unidades de cuidado neonatales (Nair, Gupta, y Jatana, 2003). Los autores plantean que esta estimulación puede ser la base de los desórdenes de integración sensorial que presentan a lo largo de su desarrollo evolutivo. Por su parte, Wickremasinghe y colaboradores (2013) plantean una explicación alternativa, al argumentar que no se debe descartar que el daño cerebral y los impedimentos en el desarrollo neurológico asociados con la prematuridad puedan ser los desencadenantes de un desorden de procesamiento sensorial.

\section{Objetivos e hipótesis}

Existen escasos estudios en España que comparen el procesamiento sensorial de niños nacidos pretérmino y a término. Por tanto, el objetivo general de esta investigación fue realizar un estudio piloto que describa las posibles disfunciones de integración sensorial de los niños nacidos pretérmino utilizando escalas de valoración específicas para la edad de los niños. Se espera que estas disfunciones estén relacionadas con la edad gestacional, y que la detección de ellas justifique y permita la intervención de estos niños por parte de un terapeuta ocupacional formado en integración sensorial, ya que mejoraría las posibles disfunciones sensoriales y el funcionamiento cotidiano del niño. Con este estudio piloto, por tanto, se intenta llenar este vacío al investigar las posibles disfunciones en el procesamiento sensorial de los niños nacidos pretérmino.

\section{MÉTOdo}

\section{Participantes}

En el presente estudio han participado 35 niños prematuros y no prematuros que, en el momento del estudio, contaban con edades comprendidas entre los 2 y 
los 4 años. Todos los niños son de nacionalidad española y ninguno de los padres pertenece a un grupo de riesgo social —entendiendo por riesgo social familias con bajos recursos socioeconómicos y socioeducativos, situaciones de exclusión social, familias desestructuradas y con conductas de consumo de sustancias- de acuerdo a la evaluación realizada en su ingreso al centro de atención temprana o al centro escolar. Los menores no presentaban ninguna patología asociada o dificultad severa en su desarrollo, más allá de las propias derivadas de la prematuridad.
La información detallada sobre los niños pretérmino y niños nacidos a término se presenta en la Tabla 1 , en la que también se diferencia entre aquellos que contestaron al cuestionario TODDLER (diseñado para los de edades comprendidas entre 7 y 35 meses) y aquellos que contestaron al cuestionario CHILD (diseñado para los niños de edades comprendidas entre los 3 y los 14 años y 11 meses).

Cabe destacar que todos los niños a término se encontraban escolarizados, mientras que todos los niños nacidos prematuramente no lo estaban, pero recibían un tratamiento de estimulación en servicios de atención temprana.

Tabla 1.

Características sociodemográficas y clínicas de los participantes.

\begin{tabular}{|c|c|c|c|c|c|c|c|c|c|}
\hline Grupo & $\begin{array}{c}\text { Total } \\
\text { participantes }\end{array}$ & $\begin{array}{l}\text { Edad crono- } \\
\text { lógica (años) } \\
\text { (media) }\end{array}$ & $\begin{array}{c}\text { Sexo } \\
\text { femenino } \\
\mathrm{n}(\%)\end{array}$ & $\begin{array}{c}\text { Edad } \\
\text { gestacional } \\
\text { media (semanas) }\end{array}$ & $\begin{array}{l}\text { Peso medio al } \\
\text { nacer (gramos) }\end{array}$ & $\begin{array}{l}\text { Tiempo me- } \\
\text { dio de ingre- } \\
\text { sos (días) }\end{array}$ & $\begin{array}{c}\text { APGAR 1 } \\
\text { minutos } \\
\text { (media) }\end{array}$ & $\begin{array}{c}\text { APGAR } 5 \\
\text { minutos } \\
\text { (media) }\end{array}$ & $\begin{array}{l}\text { Ventilación } \\
\text { asistida (\%) }\end{array}$ \\
\hline $\begin{array}{c}\text { Prematuros } \\
\text { TODDLER }\end{array}$ & 13 & 2.0 & $6(54,5 \%)$ & 27,55 & 1026,80 & 73,20 & 5,88 & 7,25 & $5(45,4 \%)$ \\
\hline $\begin{array}{c}\text { Niños nacidos a } \\
\text { término TODDLER }\end{array}$ & 10 & 2.0 & $3(30 \%)$ & 38,50 & 3135,00 & 0 & 8,78 & 9,33 & $0(0 \%)$ \\
\hline Prematuros CHILD & 5 & 3.2 & $2(40 \%)$ & 27,40 & 876,20 & 78,80 días & 4,67 & 8 & $5(100)$ \\
\hline $\begin{array}{l}\text { Niños nacidos a } \\
\text { término } C H I L D\end{array}$ & 7 & 4.0 & $1(14,2 \%)$ & 39,43 & 3358,57 & 0 & 8,43 & 9,71 & $0(0 \%)$ \\
\hline
\end{tabular}

\section{Instrumento}

Para la evaluación del perfil sensorial de ambos grupos de niños pretérmino y grupo de niños nacidos a término se han usado dos de los cuestionarios pertenecientes al SENSORY PROFILE 2 (Dunn, 2014): A) Sensory Profile 2 TODDLER Record Form 7-35 months, Spanish. B) Sensory Profile 2 CHILD Record Form 3:014:11, Spanish.

Ambos cuestionarios incluyen una lista de preguntas con las que se pretende valorar cómo responde un niño a las diferentes situaciones de la vida cotidiana. Las preguntas de este perfil sensorial tratan de identificar cómo los inputs sensoriales (visuales, auditivos, táctiles, táctiles-orales, vestibulares y propioceptivos) afectan al niño y a su desempeño en las diversas actividades de la vida diaria. El cuestionario fue facilitado y explicado por la examinadora a los padres de los menores participantes, ya que fueron ellos los que se encargaron de completar las preguntas incluidas en dicho cuestionario.

Atendiendo a las respuestas a esta serie de preguntas, se podrán observar patrones en las reacciones del niño ante las sensaciones que recibe. Se describen cuatro clases de patrones de procesamiento sensorial:

Niño evitador de sensaciones. Se trata de aquel niño al que le molestan determinadas sensaciones y las evita en todo lo posible. Suele necesitar rutinas, actividades que lo tranquilicen y presenta determinados comportamientos para evitar situaciones o actividades desconocidas. Este niño estaría en el grupo de trastornos de la modulación sensorial —revisados en la introducción-, en la categoría de hiperrespuesta. 
Niño sensitivo sensorial. Presenta mucha mas sensibilidad que otros. Es un niño hipersensible, que se distrae y dispersa con mucha facilidad y le cuesta concentrarse en las actividades con otros ni ños y la escuela. Este niño igualmente estaría en la categoría de hiperrespuesta en los trastornos de modulación sensorial.

Niño bajo registro. Se distrae y dispersa con mucha facilidad y le cuesta concentrarse en las actividades con otros niNo suele recibir el estímulo sensorial y parece no darse cuenta de lo que pasa. Se muestra menos interesado en participar en actividades y menos activo que otros niños. El/la niño/a bajo registro es el que se denomina niño/a baja respuesta, en los trastornos de modulación, en la figura propuesta por Miller y cols (2007).

Niño buscador de sensaciones. Suele buscar fuertemente el estímulo sensorial. Busca estímulos brillantes, fuertes, le gusta el movimiento, la presión en las articulaciones, saltar y girar. Este/a niño/a, igualmente se encuentra dentro de la categoría de trastornos de modulación y tendría la misma denominación, buscador sensorial.

El Sensory Profile, consiste es una batería de preguntas agrupadas en varias áreas. Y las respuestas a estas preguntas son las que nos determinan el cuadrante sensorial al que pertenece el niño valorado. Estas áreas o secciones sensoriales - auditiva, visual, táctil, y movimiento- sobre las que se indaga son comunes a ambos cuestionarios (TODDLER y CHILD).

La manera de responder a estas preguntas es otorgando una puntuación que va de 5 a 1, donde el 5 tiene un valor de "casi siempre", cuando el niño realiza la conducta sobre la que se indaga un $90 \%$ de las veces, se puntúa 4 cuando el niño realizar la conducta "frecuentemente" (un 75\% de las veces), se otorga un valor de 3 cuando el niño responde "la mitad del tiempo" (un $50 \%$ de las veces), se puntúa 2 cuando el niño realiza la conducta "ocasionalmente" (25\% de las veces) y se puntúa 1 cuando el niño realiza la conducta "casi nunca" (un $10 \%$ de las veces o menos). Existe también la posibilidad de puntuar con 0 , cuando la conducta que se describe es "no aplicable" al niño evaluado.

\section{Procedimiento}

El estudio no contó con financiación de ninguna entidad pública o privada. La examinadora invirtió un total de tres meses en toda la realización del proceso y se contó con el apoyo de un experto en metodología de las ciencias del comportamiento, para analizar los datos obtenidos.
La muestra de niños prematuros se recogieron en el centro de atención temprana donde trabaja la investigadora, esto es, el Centro de Atención e Intervención Temprana del Hospital Universitario de San Rafael, en Granada; por tanto, todos los sujetos se encontraban recibiendo algún tipo de intervención temprana (psicomotricidad, estimulación sensorial, cognitiva o del lenguaje), pero no recibían terapia de integración sensorial.

El grupo de niños nacidos a término se recogió en una escuela infantil de la misma localidad, Granada. Escuela Infantil Virgen Madre. Para ello, se pidió colaboración a la psicóloga y director del centro, y se hizo una reunión informativa a los padres en la que se les explicaba en qué consistía la investigación. Todos los padres dieron su consentimiento informado, y el estudio se realizó de acuerdo con la Ley 14/2007, de 3 de julio, de Investigación biomédica.

\section{Análisis de datos}

Dado que existe una versión del Sensory Profile para los niños de 7 a 30 meses (TODDLER), y otra para los niños de 3 a 14 años (CHILD), se decidió corregir los puntajes de dicho instrumento de forma separada para cada uno de los dos grupos.

Primero, para cada grupo de niños se calculó, siguiendo las pautas establecidas por Dunn (2014), el sumatorio de los ítems relacionados con cada uno de los cuatro cuadrantes sensoriales (Buscador, Evitador, Sensitivo, Bajo Registro), y de las cinco secciones sensoriales que ambos instrumentos tienen en común (visual, táctil, auditivo, oral, y movimiento). Esto es lo que se denominará en este artículo como "las puntuaciones brutas".

Segundo, utilizando las puntuaciones brutas, y siguiendo los criterios establecidos por el sistema de clasificación del Sensory Profile 2, se asignó a los participantes a uno de los tres siguientes grupos de desarrollo: 1) Menor que el desarrollo normativo, 2) Igual que la mayoría de los demás (grupo de desarrollo normativo), 3) Mayor que el desarrollo normativo.

Tercero, se estandarizaron las puntuaciones brutas; es decir, se obtuvieron puntuaciones $Z$. Las puntuaciones $Z$ (que calculan la distancia a la que cada participante se encuentra de la media de su grupo de referencia) permiten integrar en un mismo análisis estadístico a los dos grupos (niños de 7 a 30 meses (TODDLER) y niños de 3 a 14 años (CHILD)). 
Dado que el tamaño de cada uno de los grupos de edad es pequeño, impide realizar un contraste estadístico entre todos los subgrupos establecidos en la metodología, todos los análisis se realizaron incluyendo a dos grupos: niños de 7 a 30 meses (TODDLER) y niños de 3 a 14 años (CHILD).

En concreto, se realizaron dos análisis estadísticos —utilizando el programa SPSS-. Primero, para examinar si existían diferencias en las puntuaciones brutas de los niños prétermino y de los niños a término, se realizaron varias pruebas $t$ para muestras independientes en las que introdujo como variable independiente el grupo de edad gestacional (prétermino vs. a término) y como variables dependientes las puntuaciones brutas (transformadas a puntuaciones Z) de las distintas dimensiones medidas por el Sensory Profile2. Segundo, se comparó el porcentaje de niños a término y de niños pretérmino que pertenecen al grupo de desarrollo normativo en cada una de las dimensiones del Sensory Profile 2, a través de varias pruebas de chi cuadrado.

\section{Resultados}

\section{Comparación del porcentaje de niños dentro del grupo de desarrollo normativo}

Diversos análisis de chi cuadrado compararon el porcentaje de niños a término y de niños pretérmino que se encontraban dentro del grupo que, siguiendo los criterios establecidos por el Sensory Profile2 (Dunn, 2014), puede considerarse como el grupo de desarrollo normativo. Este análisis se realizó para cada uno de los cuadrantes sensoriales (Tabla 2) y para las cinco secciones sensoriales (Tabla 3). Como se puede ver en las Tablas 2-3, en ninguno de los casos se encontraron diferencias significativas.

\section{Comparación de las puntuaciones brutas}

Como se ha indicado anteriormente, para comparar las diferencias entre niños a término y niños prétermino en las puntuaciones brutas del Sensory Profile2, se realizaron varias pruebas t para muestras independientes.

En relación a los cuatro cuadrantes sensoriales, y como se puede ver en la Tabla 4, sólo se encontraron diferencias significativas en el estilo sensorial Bajo Registro, en el que los niños pretérmino puntuaron más alto que los niños a término, $t(31)=2.82 ; p=.008 ; d$ $=1.01$.

Por otro lado, y como se observa en la Tabla 5, se encontraron diferencias significativas entre ambos grupos en las secciones sensoriales táctil $(t(31)=2.91 ; p$ $=.007 ; d=1.04), y$ de movimiento $(t(31)=2.79 ; p=$ $.009 ; d=1.00)$, en las que, nuevamente, los niños pretérmino puntuaron más alto.

Tabla 2.

Porcentaje de niños que pertenecen al grupo normativo en función de la edad gestacional (pretérmino vs. a término) y del perfil sensorial.

\begin{tabular}{|c|c|c|c|}
\hline \multirow{2}{*}{ PERFIL } & NIÑOS PRETÉRMINO & $\begin{array}{c}\text { NIÑOS A } \\
\text { TÉRMINO }\end{array}$ & $p$ \\
\hline BUSCADOR & $75.0 \%$ & $76.5 \%$ & .92 \\
\hline EVITADOR & $68.8 \%$ & $70.6 \%$ & .91 \\
\hline SENSITIVO & $75.0 \%$ & $82.4 \%$ & .61 \\
\hline BAJO REGISTRO & $81.3 \%$ & $76.5 \%$ & .73 \\
\hline
\end{tabular}


Tabla 3.

Porcentaje de niños que pertenecen al grupo normativo en función de la edad gestacional (pretérmino vs. a término) y de la sección sensorial.

\begin{tabular}{|c|c|c|c|}
\hline SECCIÓN & NIÑOS PRETÉRMINO & NIÑOS A TÉRMINO & $p$ \\
\hline AUDITIVO & $81.3 \%$ & $76.5 \%$ & .73 \\
VISUAL & $87.5 \%$ & $94.1 \%$ & .51 \\
TACTIL & $68.8 \%$ & $70.6 \%$ & .90 \\
ORAL & $62.5 \%$ & $76.5 \%$ & .38 \\
MOVIMIENTO & $68.8 \%$ & $76.5 \%$ & .62 \\
\hline
\end{tabular}

Tabla 4.

Puntuaciones brutas (Z) en función de la edad gestacional (pretérmino vs. a término) y del perfil sensorial.

\begin{tabular}{|c|c|c|c|}
\hline PERFIL & NIÑOS PRETÉRMINO & NIÑOS A TÉRMINO & $p$ \\
\hline BUSCADOR & .10 & -.09 & .57 \\
\hline EVITADOR & .10 & -.10 & .57 \\
\hline SENSITIVO & .32 & -.30 & .06 \\
\hline BAJO REGISTRO & .45 & -.42 & $<.01^{*}$ \\
\hline
\end{tabular}

Tabla 5.

Puntuaciones brutas $(Z)$ de niños que pertenecen al grupo normativo en función de la edad gestacional (pretérmino vs. a término) y de la sección sensorial.

\begin{tabular}{|c|c|c|c|}
\hline SECCIÓN & NIÑOS PRETÉRMINO & $\begin{array}{c}\text { NIÑOS A } \\
\text { TÉRMINO }\end{array}$ & $p$ \\
\hline AUDITIVO & .30 & -.29 & .08 \\
\hline VISUAL & -.30 & .28 & .09 \\
\hline TACTIL & .46 & -.43 & $<.01 *$ \\
\hline ORAL & .16 & -.15 & .35 \\
\hline MOVIMIENTO & .44 & -.42 & $<.01^{*}$ \\
\hline
\end{tabular}

\section{DisCUSIÓN}

Atendiendo a los resultados obtenidos en la presente investigación, no se han encontrado diferencias significativas entre los niños prematuros y niños a término en cuanto al porcentaje de niños que pertenecían al grupo normativo. Pero sí hubo diferencias en los puntajes brutos; es decir, aunque las puntuaciones de ambos grupos se encontraron dentro de los valores considerados como valores norma en el Sensory Profile, podemos destacar que las puntuaciones de los niños prematuros presentan una tendencia a estar por encima de las del grupo control, y esto es significativo en el cuadrante sensorial de Bajo Registro y en los estilos sensoriales de movimiento y tacto. Estos resultados encontrados son similares a los que nos proporciona la literatura, si no en porcentajes si en cuanto a los cuadrantes y estilos sensoriales (Rahkonen et al., 2015; Wickremasinghe et al., 2013). 
Estos resultados son destacables en la medida en que justifican la intervención de todos los niños nacidos prematuramente, hayan padecido o no patologías asociadas al hecho de haber nacido de manera prematura (Naberhaus y Segarra, 2004). Y más allá de esto, justifican la intervención de esta población por parte de un terapeuta ocupacional, formado en integración sensorial, profesional que hoy en día no forma parte del equipo base de la atención temprana. Estos resultados pueden animar al conocimiento y difusión de esta terapia tan extendida ya en otros sitios del mundo, y que en los centros públicos de intervención en España apenas está comenzando a conocerse. Así, mientras que "en países como Australia, Canadá, Estados Unidos o Suecia, los tratamientos de integración sensorial son habituales desde hace varias décadas, tanto en el ámbito sanitario como en el escolar, para remediar estos problemas, pero por desgracia en España aún son muy pocos los niños que se benefician de dicho enfoque terapéutico. El País Vasco es la única comunidad autónoma española que ya ofrece a los escolares servicios de Terapia Ocupacional Pediátrica, dentro de los cuales se incluyen tratamientos basados en la teoría de la integración sensorial" (Beaudry-Bellefeuille, s.f., párrafo 18).

El papel del terapeuta ocupacional se considera imprescindible dentro del equipo de atención temprana por la visión global que este profesional tiene de la niñez, de los componentes de ejecución que están afectados en la realización de las diferentes actividades y por su capacidad para analizar y adaptar dichas actividades para que el/la niño/a sea capaz de realizarlas de la manera más funcional posible.

Por su formación académica de base, el terapeuta ocupacional que trabaja en pediatría es capaz de evaluar e intervenir en las áreas del desempeño ocupacional como son la motora - tanto a nivel motor grueso como manipulativo-, la perceptiva, o la autonomía en actividades de la vida diaria; esto es, en el juego, actividades productivas escolares, el vestido o la alimentación.

El terapeuta ocupacional pediátrico que además posee formación en integración sensorial tiene una visión aún más rica del niño en su conjunto, y más herramientas que le ayudan a explicar el porqué de muchas de las manifestaciones comportamentales que se suelen observar, y que son interpretadas por otros profesionales como problemas de conducta o de atención (lo que podría llevar a tratamientos que en muchas ocasiones no surten el efecto deseado).
Por todo esto, es necesario encontrar la forma de introducir a este profesional dentro del equipo multidisciplinar que forma el equipo de atención temprana, e investigaciones de este tipo pueden respaldar y justificar el papel del terapeuta ocupacional en estos dispositivos.

En cuanto a las limitaciones que nos hemos encontrado a la hora de realizar este trabajo y que probablemente han influido en los resultados obtenidos en el mismo, podemos apuntar los siguientes. En primer lugar, el tamaño de la muestra, ya que consideramos que ha sido demasiado pequeña como para arrojar resultados concluyentes. Según los datos del Instituto Nacional de Estadística (INE) cada año se producen es España alrededor de 29.000 partos prematuros, un porcentaje del $6.4 \%$, lo que supone 1 de cada 13 nacimientos. De estos, según la misma fuente, unos 708 partos prematuros han ocurrido en Granada en 2014. Por tanto, la muestra utilizada en este estudio se encuentra lejos de llegar a ser representativa de la población estudiada.

Otro posible escollo es que todos los niños pretérmino estaban siendo atendidos en un centro de atención temprana, y aunque no estaban recibiendo terapia de integración sensorial, si estaban recibiendo otro tipo de intervenciones que influyen en sus respuestas conductuales y comportamentales. Esto podría explicar que un alto porcentaje de los niños prétermino mostrasen puntuaciones normativas en los distintos cuadrantes del Sensory Profile.

En lo relativo al cuestionario en sí, también encontramos alguna dificultad, ya que algunas preguntas del cuestionario, en opinión de los padres, han sido difíciles de responder. Asimismo, se plantea la necesidad de comparar ambos grupos de población no solo mediante el presente cuestionario, si no mediante el uso de otras pruebas estandarizadas observables por el propio evaluador como el Test of Sensory Functions in Infants (DeGangi y Greenspan, 1989) o el Bayley Scales of Infants and Toddler Development III (Bayley, 2005), que no han sido utilizados en esta ocasión ya que se trataba de un estudio preliminar y no se contó con el tiempo suficiente como para realizar una evaluación exhaustiva.

A pesar de sus limitaciones, creemos que el presente trabajo es un primer paso para examinar en una muestra española las disfunciones en integración sensorial mostrada por los niños prematuros, y por tanto concienciar sobre las distintas dificultades que suele enfrentar este colectivo. 


\section{ReferenCias Bibliográficas}

AAVV. (2000). Libro Blanco de la Atención Temprana. Madrid: Real Patronato de Prevención y de Atención a Personas con Minusvalía.

Asociación Profesional de Terapeutas Ocupacionales de Navarra. (2004). Terapia Ocupacional en Atención Temprana. Recuperado de Terapia-Ocupacional.com

Ayres, A.J. (1978). Sensory Integration and Child. Los Ángeles, CA: Western Psychological Services.

Ayres, J. (2008). La Integración Sensorial en los niños. Desafíos sensoriales ocultos. Ed. 25 aniversario. Madrid: TEA.

Asociación Española de Integración Sensorial (s.f.). Recuperado de http:// www.integraciónsensorial.es

Bart, O., Shayevits, S., Gabis, L. V., y Morag, I. (2011). Prediction of participation and sensory modulation of late preterm infants at 12 months: A prospective study. Research in developmental disabilities, 32, 2732-2738.

Bayley N. (2005). Bayley Scales of Infants and Toddler Development-III. Bloomington, Mn: Pearson.

Beaudry Bellefeuille I. (2013). El enfoque de la integración sensorial de la doctora Ayres. Revista de Terapia Ocupacional Gallega, 10, 1-11.

Beaudry Bellefeuille I. (s.f.). Portal de Terapia Ocupacional Pediátrica. Recuperado de http://www.ibeaudry.com/s5/integracion-sensorial/

DeGangi G. y Greenspan S. (1989) Test of Sensory Functions in Infants. Torrance, CA: WPS.

Domínguez Jiménez, I., Calvo Arenillas, JL. (2015). Terapia Ocupacional y su papel en atención temprana: revisión sistemática. TOG, 12, 1-22.

Dunn, W. (2014). Sensory Profile 2 (Spanish). Bloomington, Mn: Pearson.

Graven, S. N., y Browne, J. V. (2008). Sensory development in the fetus, neonate, and infant: introduction and overview. Newborn and Infant Nursing Reviews, 8, 169-172. doi: 10.1053/j.nainr.2008.10.007

Imperatore Blanche, E. (2005). Déficit de Integración Sensorial: Efectos a largo plazo sobre la ocupación y el juego. Revista chilena de terapia ocupacional, 5, 1-6.

Miller, L. J., Anzalone, M. E., Lane, S. J., Cermak, S. A., y Osten, E. T. (2007). Concept evolution in sensory integration: a proposed nosology for diagnosis. The American Journal of Occupational Therapy, 61, 135-140. doi: 10.5014/ajot.2015.013755

Mitchell, A. W., Moore, E. M., Roberts, E. J., Hachtel, K. W., y Brown, M. S. (2015). Sensory Processing Disorder in Children Ages Birth-3 Years Born Prematurely: A Systematic Review. The American Journal of Occupational Therapy, 69, 1-11. doi: 10.5014/ajot.2015.013755

Moral, G., Pastor, MA., y Sanz, P. (2013). Del Marco Teórico de Integración Sensorial al modelo clínico de intervención. TOG, 10, 1-25.

Naberhaus, A., Segarra, D. (2004). Trastornos neuropsicológicos y del neurodesarrollo en el prematuro. Anales de Pediatría, 20, 317-326.

Nair, M. N. G., Gupta, G., y Jatana, S. K. (2003). NICU environment: Can we be ignorant? Medical Journal Armed Forces India, 59, 93-95. doi: 10.1016/S0377-1237(03)80046-1
Rahkonen, P., Lano, A., Pesonen, A.K., Heinonen, K., Räikkönen, K., Vanhatalo, S.,Autti, T., Valanne, L., Andersson, S. and Metsäranta, M. (2015), Atypical sensory processing is common in extremely low gestational age children. Acta Paediatrica, 104, 522-528. doi:10.1111/ apa.12911

Wickremasinghe, A., Rogers, E., Johnson, B., Shen, A., Barkovich, A., y Marco, E. (2013). Children born prematurely have atypical Sensory Profiles. Journal of perinatology: Official journal of the California Perinatal Association, 33, 631-635. doi: 10.1038/jp.2013.12 